\title{
Evaluation of Root Canal Filling with a Bioceramic Sealer Using Micro-Computed Tomography: A Pilot Study
}

\author{
Isabel Vasconcelos ${ }^{1, a^{*}}$, Mário Rito Pereira ${ }^{1, b}$, António Ginjeira ${ }^{1, c}$, \\ Margarida Franco ${ }^{2, \mathrm{~d}}$, Pedro Morouço ${ }^{2, \mathrm{e}}$, Nuno Alves ${ }^{2, \mathrm{f}}$
}

${ }^{1}$ Department of Endodontics, School of Dental Medicine, University of Lisbon, Portugal

${ }^{2}$ Centre for Rapid and Sustainable Product Development, Polytechnic Institute of Leiria, Portugal

aisabelvascon@hotmail.com, bmariorp@me.com, caginjeira@campus.ul.pt, dmargarida.franco@ipleiria.pt, eppedro.morouco@ipleiria.pt, fnuno.alves@ipleiria.pt

${ }^{*}$ corresponding author

Keywords: root canal filling, bioceramic sealer, micro-CT

\begin{abstract}
This pilot study aims to define a protocol for optimizing the micro-computed tomography (micro-CT) settings to evaluate in the future research the root canal filling in oval shaped canals. Thirty distal canals of mandibular molars were used. After preparation of the canals, the roots were randomly divided into five groups. The canals were filled with two types of sealers (Endosequence BC Sealer or AH Plus) and two types of gutta percha (Protaper Next cones and Endosequence BC cones), using thermal obturation in comparison with a single cone obturation technique. Each specimen was scanned three times using a micro-CT device at a resolution of 30,1 $\mu \mathrm{m}$. The first scan was done for selecting the specimen according the inclusion criteria, the second one postinstrumentation and the last micro-CT scan after obturation the specimens.

For the present study, the images were only evaluated with qualitative criteria and the settings for acquisition, reconstruction and analysis of micro-CT images were tested.
\end{abstract}

\section{Introduction}

Complete sealing of the root canal system after cleaning and shaping is critical to prevent oral pathogens from colonizing and re-infecting the root canal system and periapical tissues [1]. During this process, the anatomy of the root canal system should always be taken into account [2]. Despite numerous variations, certain typical canal configurations have been described. Differences in the cross-section of root canals are related to tooth types. There are teeth with relatively round root canal cross-sections but we also have oval, long oval, flattened or irregular counterparts, typically observed in distal roots of mandibular molars [2]. Several studies have shown that irrespective of the preparation technique used, the instruments were not able to remove the inner layer of dentine during preparation of long oval root canals [3]. Furthermore, even when using thermoplasticized guttapercha techniques, there is limited ability to completely fill oval shaped root canals [4,5].

Because it is not possible to sterilize the canal or remove all debris during instrumentation, the goal of obturation is to prevent coronal leakage, prevent influx of periapical fluids and entomb remaining bacteria in the root canal [6]. Microleakage results from voids occurring at the interface of the dentinal wall and root filling material or through voids within the filling material [7]. Therefore, the smaller the number of gap-containing regions, the better the performance of the root filling tends to be. It means that one of the goals of the filling procedure must be to maximize voids-free regions [8]. So, the quality of root canal filling and coronal restoration after root canal treatment has a strong effect on the long-term outcome of endodontic treatment [9].

One of the most important components of root filling is the root canal sealer. Endodontic sealers play an important role in filling root canal spaces. Indeed, root filling will leak in the absence of a sealer [10]. However, the assumption that the sealer creates a coating barrier between gutta-percha and dentin may be an incorrect assumption because the majority of sealers has shrinkage during setting time and do not have dimensional stability over the time [11]. Ideally, the sealer should be 
capable of producing a bond between core material and dentine wall because gutta-percha does not directly bond to the dentin surface [12].

Accordingly, new materials have been developed over the past years. A variety of sealers have been employed in root canal filing, attempting to provide an ideal root canal sealer, that should be biocompatible, antibacterial, nontoxic and radiopaque. Furthermore, it should also be fluid-tight, dimensionally stable, and adhere effectively to the root canal wall.

Systematic research of ceramics for use in biomedical applications began in the early of seventies, and over the past forty years, the application of a variety of ceramics in biomedicine has greatly expanded. Bioceramics have also been introduced in dentistry and endodontic researchers claims interest in bioceramic technology mainly related to physical and biological properties. According to manufacturers, bioceramics are extraordinarily biocompatible and they are chemically stable within the biological environment. Also, bioceramics do not shrink upon setting. In fact, they actually expand slightly upon completion of the setting process. A further advantage of the material itself is its ability (during the setting process) to form hydroxyapatite and ultimately establish a chemical bond between dentin, witch has a mineral phase- hydroxiapatite [13] and gutta percha, especially with bioceramic particles. Another advantage is its high $\mathrm{pH}$ (12.8) during the initial 24 hours of the setting process (which is strongly antibacterial), its excellent sealing ability, and its ease of use (particle size is small enough to be used in a syringe with a cappilary tip). The use of bioceramic-based sealers with their features- osseoconductivity, hydrophilicity, adhesiveness and chemical bonding to the root canal dentinal walls appears to be an effective approach to eliminate on long-term, the micro space, otherwise remaining between the root canal walls and other kinds of sealers [14].

Studies have shown the advantages of using thermoplastic obturation techniques, mainly in oval or irregular root canals [15]. The single-cone technique, which is becoming increasingly popular, uses gutta-percha cones with the diameter and taper matched to the final shape of canals given by the mechanized instrumentation system used. The advantages of this technique include easy handling, low cost, and short procedure time [16, 17]. Among the disadvantages of this approach, the possibility of porosities when dealing with large volumes of sealer, mainly the traditional ones with setting contraction and material dissolution are worth noting.

The main objective for the present study was to test a protocol for the specimen preparation and acquisition of the micro-CT images to evaluate the quality of obturation by determining the volume of filling and the voids and to determine which of the materials and filling techniques are able to obturate the root canals with less voids. Several microscopy techniques are currently used to evaluate the root canal filling, including stereomicroscopy, scanning electron microscopy (SEM), transmission electron microscopy (TEM) and confocal laser scanning microscopy $[18,19]$. However, the main disadvantage of these techniques is the potential for producing artifacts during specimen preparation but also during examination in a high vacuum environment. Micro- CT is a non-destructive threedimensional (3D) technique. Micro-CT applied to endodontics allows 3D assessment of the obturation quality, permitting to identify areas of failure and voids. In addition, it provide the quantification of the filling material volume present in the canal, with is an important advantage for preserving the specimen [20,21]. A massive amount of information may be generated from scans; slices may be recreated in any plane and data may be represented as two-dimensional or 3D images and evaluated qualitatively or quantitatively.

There are several available software's for 3D reconstruction of the generated images from the micro-CT. Generally, these are either expensive, with limited availability and might not totally serve our purposes in the endodontic field. MeVisLab (MeVis Research, Bremen, Germany) is an opensource software that uses a framework system for medical image processing and visualization. Besides being a freely available platform, it allows the creation of a user-oriented application frameworks and the design of user interfaces with MDLscript (MeVis Description Language), reducing the complexity of user interface programming from non-technical users (Fig.1). 


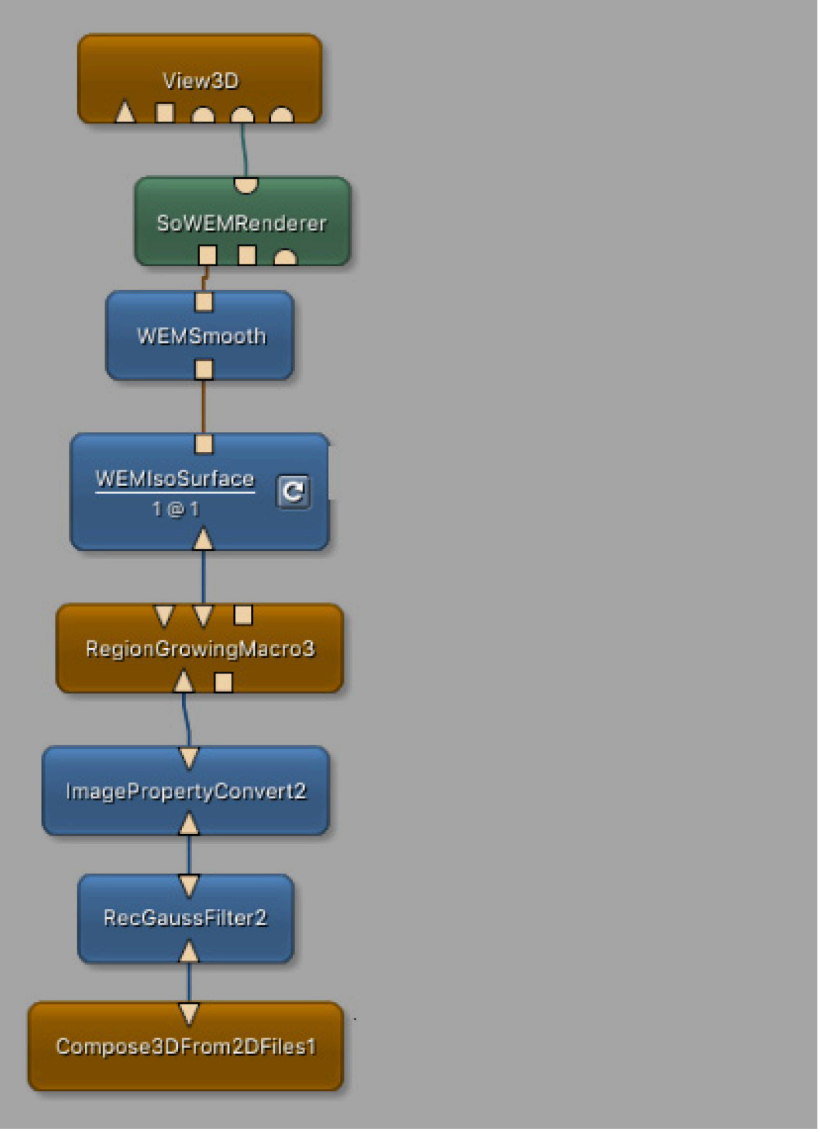

Fig. 1 - Shows MeVisLab framework used to 3D reconstruct the roots of a lower molar with a small amount of modules available in MeVisLab: a module for loading the image data, an algorithm module, which applies a threshold function in this example, and a viewer module for the visualization.

The application framework based on MeVisLab enables a standardized method of 3D reconstruction and measurements of canal and teeth scanned by micro-CT. This provides powerful and promising tools widely available for solving 3D problems in contemporary endodontic research.

\section{Materials and Methods}

For the fillings a widely used sealer - AH Plus (Dentsply DeTrey, GmbH, Konstanz, Germany), was compared with a recent bioceramic sealer - EndoSequence BC Sealer (Brasseler USA, Savannah, GA, USA), using two techniques: cold (single cone) and a thermoplastic technique (continuous wave).

Sample selection. A total of fifty mandibular human molars were pre operatively scanned to permit the selection of thirty teeth according to inclusion and exclusion criteria.

The defined inclusion criteria was: teeth with two roots, distal canals with Vertucci type I configuration, straight root canal, intact roots and teeth with similar length $(20 \pm 2 \mathrm{~mm})$ and distal roots with oval shaped canals. The canals were classified as oval if the buccolingual diameter of the root was 1,6 times greater its mesiodistal diameter at a level of $5 \mathrm{~mm}$ from the root apex.

The adopted exclusion criteria was: roots with resorptive defects, cracks or open apices, apical gauges above $0,40 \mathrm{~mm}$, teeth with previous endodontic fillings.

Specimen preparation. The crown of each tooth was cut at the cementoenamel junction to standardize the canal length at $12 \mathrm{~mm}$. Rotary instrumentation was carried out with the Protaper Next system (Dentsply Maillefer, Ballaigues, Switzerland) following the sequence X1, X2, X3 and X4 in a X Smart motor (Dentsply-Maillefer, Ballaigues, Switzerland), at $300 \mathrm{rpm}$ and $2 \mathrm{~N} / \mathrm{cm}$. The instrumentation was performed until an apical diameter of 40.06 [8]. Roots with apical diameter above $0.40 \mathrm{~mm}$ were discarded. 
Between each instrument, the canal was irrigated with $2 \mathrm{~mL}$ of a freshly prepared solution of $5,25 \%$ sodium hipochlorite $(\mathrm{NaOCl})$ with a $30-\mathrm{G}$ side-vented needle (Endoneedle G30; Elsodent, Cergy-Pontoise, France) [22]. The needle was positioned inside a prepared root canal at $2 \mathrm{~mm}$ short of the working length (WL) [23] and apical patency was maintained with a $10 \mathrm{~K}$ file [24]. A final irrigation protocol consisted of $2 \mathrm{ml}$ of $\mathrm{NaOCl} 5,25 \%$ passively activated with ultrasound for $1 \mathrm{~min}$ (20 s, 3 times). So, the total volume of irrigant was $6 \mathrm{ml} \mathrm{[25].}$

The irrigant was energized with an ultrasonic tip ISO 20 25mm, (IrriSafe; Satelec, Actaeon Group, Merignac Cedex, France) positioned at $1 \mathrm{~mm}$ short of WL [26], by a piezoelectronic unit (Suprasson PMax; Satelec Acteon) at power setting "blue"' 4 (30 kHz).

Then, the smear layer was removed by $1 \mathrm{ml}$ of $17 \%$ EDTA for $1 \mathrm{~min}$, followed by $2 \mathrm{ml}$ of 5,25\% $\mathrm{NaOCl}$ [22] and finally each canal was washed with $2 \mathrm{~mL}$ of distilled water. The canals were dried with absorbent X4 paper points (Dentsply Maillefer) and randomized into five groups. Two different sealers, prepared according to the manufacturers' instructions were used to fill the roots canals. AH Plus Jet Sealer (Dentsply DeTrey GmbH, Konstanz, Germany) were used in groups 1 and 2 and EndoSequence BC Sealer (ES) (Brasseler, USA) in groups 3, 4 and 5.

In groups 1, 2, 3 and 5 were used Protaper X4 cones (Dentsply, Maillefer) and in group 4 were used a bioceramic gutta-percha- Endosequence BC points (40.06). In groups 1 and 5 was performed a thermoplastizied technique and in groups 2, 3 and 4 was performed a single cone technique.

One radiograph was taken to control the obturation and after obturation of the root canals, all specimens were stored in a humid atmosphere, at $37^{\circ} \mathrm{C}$, for 10 days to allow sealers to set [27].

Micro-CT Imaging. Teeth were scanned preoperatively, to investigate anatomic features, after instrumentation and obturation. The teeth were mounted on a stub and scanned in a micro-CT device (SkyScan 1174v2; Bruker-microCT) at $50 \mathrm{kV}, 800 \mu \mathrm{A}$, image pixel size of $30,1 \mu \mathrm{m}, 360^{\circ}$ around the vertical axis, rotation step of 0.9 , and using a $0,5 \mathrm{~mm}$ thick aluminum filter. The images of each specimen were reconstructed from the apex to the level of the cementoenamel junction using NRecon v.1.7.0.4 software (Bruker-microCT). The same parameters were used for pre-preparation, postpreparation and post obturation images acquisition. In a definitive protocol it will be calculated the percentage of voids by subtraction analysis between the post-instrumentation and the post-obturation area. Difference will correspond to the unfilled area.

\section{Results and Discussion}

There is limited information in the current literature on the standardization of important parameters, which must be selected before the micro-CT examination, such as data acquisition and reconstruction, and the influence of these parameters for the voids assessment. This may lead to inaccurate measurements (area and volume of fillings and voids), compromising the scientific impact of the findings. Thus, an accurate reconstruction of the root and root canal system is essential for the best qualitative results possible. An example that illustrates the importance to customize some processes, in order to obtain the most accurate reconstruction of images, is related to the choice of threshold. When using MeVisLab, if a threshold is chosen for the entire root, 3D images obtained are either not accurate in the coronal third and more precise in the apical third. This fact also occurs if the threshold is to high and/or if the interval is to large, or vice versa (Fig. 2). 


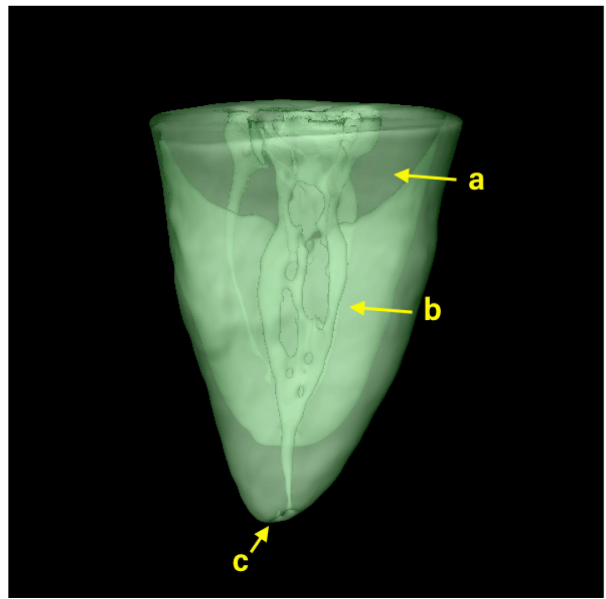

Fig. 2 - 3D Reconstruction of a root with an automatic threshold computation with a threshold interval size of $30.000 \%$.

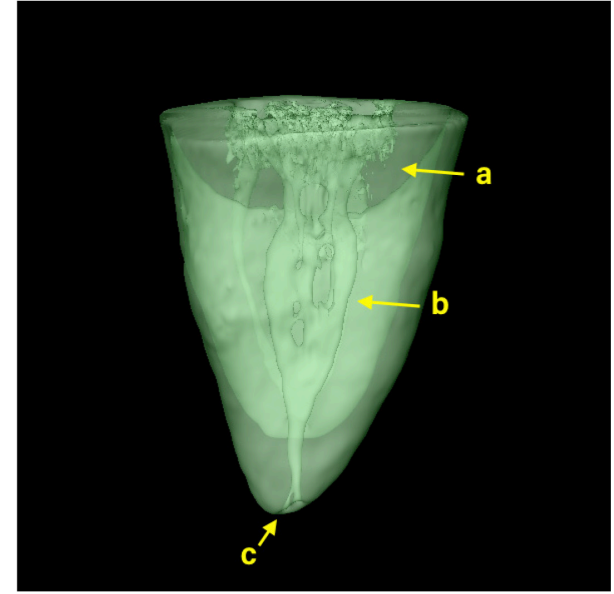

Fig. 3 - 3D Reconstruction of the same root as Fig. 2 with an automatic threshold computation with a threshold interval size of $15.000 \%$.

When a higher interval size is used the coronal third (a) of the root reconstruction is more accurate regarding its anatomy, while the middle (b) and apical (c) third lacks accuracy. On the other hand, reducing the interval size percentage of the threshold improves the reconstruction of the apical and middle third (note the presence of an apical delta and a root canal area reconstructed more precise).

This difference might occur due to the different composition along the root of the diameter and number of dentinal tubules, since in the coronal third they are larger in diameter and higher in number but decreasing in either diameter and in number to the apical third [28, 29]. This difference in the dentin composition leads to a bigger interval of greys in the coronal third and to a smaller one in the apical third.

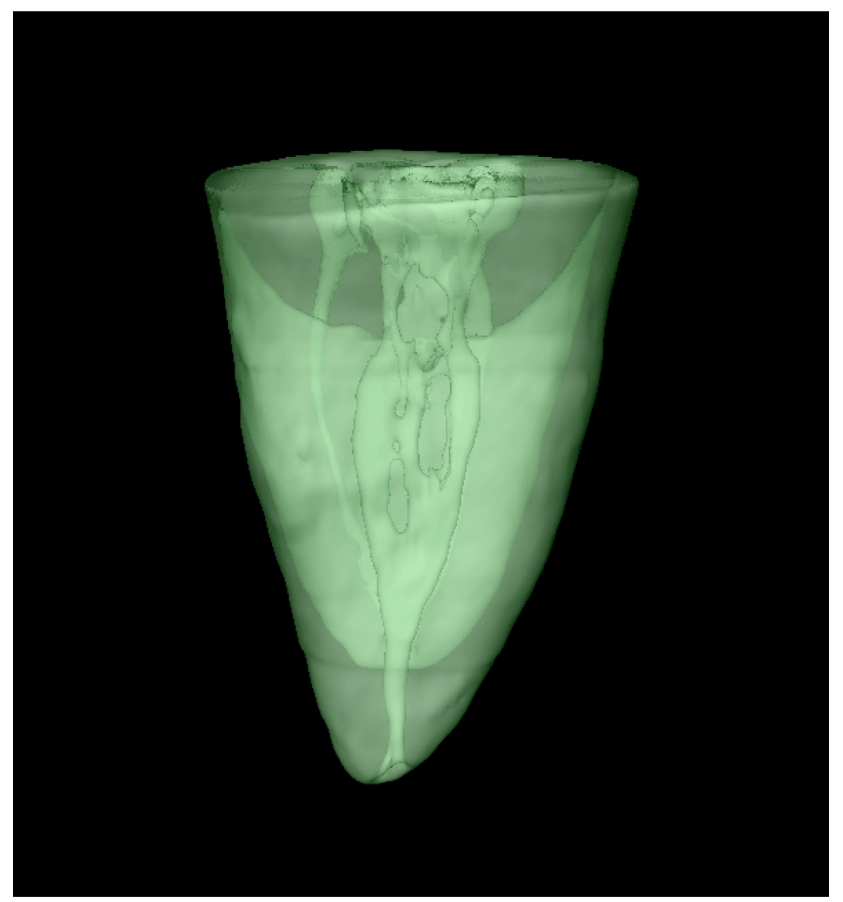

Fig. 4a

Fig. 4a - Using Mevislab, we can use the modules to apply three different thresholds interval sizes to the three thirds of the root (coronal, middle and apical) to achieve more accurate qualitative reconstruction. (Fig. 4a and $4 b$ ). 


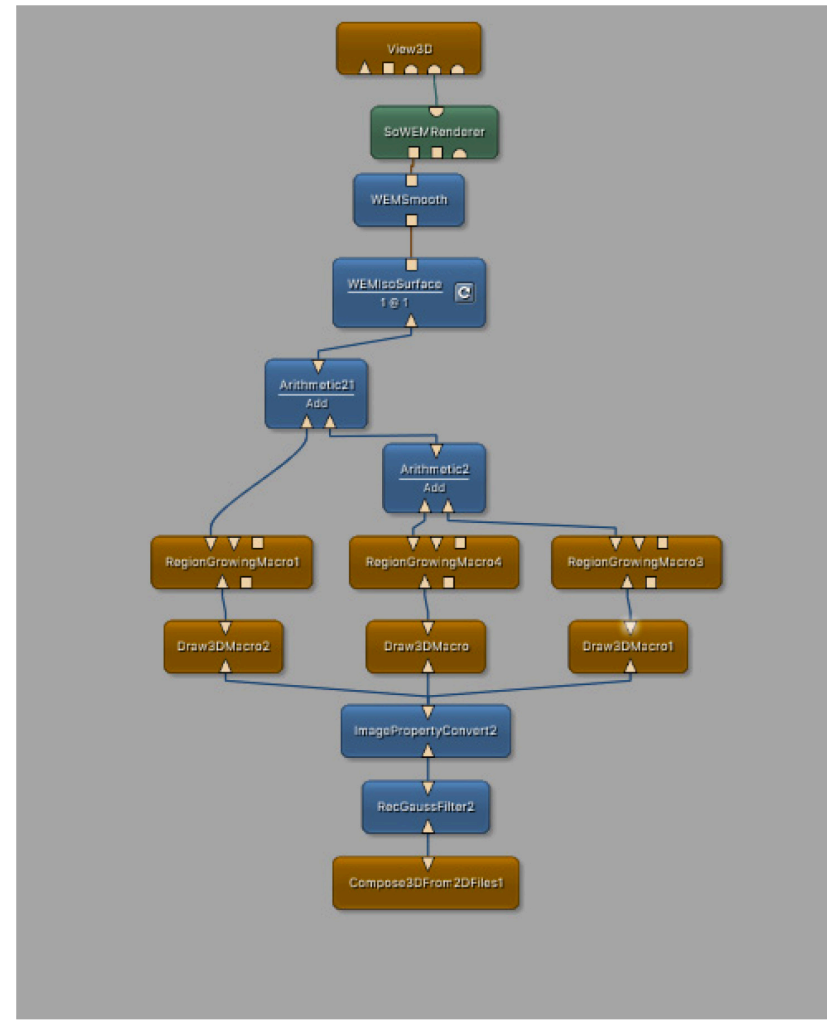

Fig. 4b - Framework used for applying three different thresholds intervals to do the 3D reconstruction at the same root.

\section{Conclusion}

The use of micro-CT in endodontics has brought several advantages for research, either for evaluation of anatomical features or treatment effects and materials used in endodontics. While acquiring images and processing them, several settings should be considered to obtain the best results with no interferences. The lack of information in current literature, mainly at the level of 3D reconstruction of the image projections obtained from micro-CT scans makes it difficult to implement protocols and improvements that can be made in this step to obtain valid and accurate both qualitatively and quantitatively results. Therefore, this pilot study revealed to be relevant because the settings for acquisition, reconstruction and analysis of micro-CT images could be tested and standardized to avoid inaccurate measurements in future research works when area post instrumentation, volume and voids of fillings are analyzed.

\section{Acknowledgements}

The Endosequence BC Sealer used in this study was kindly donated by Brasseler USA.

The AH Plus used in this study was kindly donated by Dentsply. This work was also supported by the Fundação para a Ciência e a Tecnologia (FCT) and Centro2020 through the Project references: UID/Multi/04044/2013; PAMI - ROTEIRO/0328/2013 (No 022158)

\section{Conflict of interest}

The authors have stated explicitly that there is no conflict of interest in connection with this article. 


\section{References}

[1] Bouillaguet S, Shaw L, Barthelemy J, Krejci I, Wataha JC. Long-term sealing ability of Pulp Canal Sealer, AH-Plus, GuttaFlow and Epiphany. Int Endod J 41 (2008) 219-26.

[2] Vertucci FJ. Root canal anatomy of the human permanente teeth. Oral Surgery, Oral Medicine, Oral Pathology 58 (1984) 589-99.

[3] Paqué F, Balmer M, Attin T, Peters OA. Preparation of oval-shaped root canals in mandibular molars using nickel-titanium rotary instruments: a micro-computed tomography study. Journal of Endodontics 36 (2010) 703-7.

[4] Wu M-K, Wesselink PR. A primary observation on the preparation and obturation of oval canals. International Endodontic Journal 34 (2001) 137-41.

[5] De-Deus G, Reis C, Beznos D, de Abranches AM, Coutinho- Filho T, Paciornik S. Limited ability of three commonly used thermoplasticized gutta-percha techniques in filling oval-shaped canals. Journal of Endodontics 34 (2008)1401-5.

[6] Gharib SR, Tordik PA, Imamura GM, Baginski TA, Goodell GG. A confocal laser scanning microscope investigation of the epiphany obturation system. J Endod 33 (2007) 957-61.

[7] Wu M-K, Wesselink PR. Endodontic leakage studies reconsidered. Part 1. Methodology, application and relevance. Int Endod J 26 (1993) 37- 43.

[8] DeDeus G, Reis C, Giorgi K, Brandão MC, Audi C, Fidel R. Interfacial adaptation of the Epiphany self-adhesive sealer to root dentin. Oral Surg Oral Med Oral Pathol Oral Radiol Endod 111 (2011) 381-386.

[9] Ray HA, Trope M. Periapical status of endodontically treated teeth in relation to the technical quality of the root filling and the coronal restoration. Int Endod J 28 (1995) 12-8.

[10] Kontakiotis EG, Wu MK, Wesselink PR. Effect of sealer thickness on long-term sealing ability: a 2-year follow-up study. Int Endod J 30 (1997) 307-12.

[11] Trope M, Bunes A, Debelian G. Endod Top 32 (2015) 86-96.

[12] Skinner RL, Himel VT. The sealing ability of injection molded thermoplasticized gutta-percha with and without the use of sealers. Journal of Endodontics 13 (1987) 315-7.

[13] Tjaderhane L, Carrilho M, Breschi L, Tay F, Pashley D. Dentin basic structure and composition — an overview. Endod Top 20 (2009) 3-29.

[14] Kossev D, Stefanov V. Ceramics-based sealers as new alternative to currently used endodontic sealers. Roots. 1 (2009) 42-48.

[15] Celikten B, Uzuntas CF, Orhan AI, Orhan K, Tufenkci P, Kursun S, Demiralp KO. Evaluation of root canal sealer filling quality using a single-cone technique in oval shaped canals: an in vitro Micro-CT study. Scanning 38 (2016) 133-40.

[16] Tasdemir T, Yesilyurt C, Ceyhanli KT, Celik D, Er K. Evaluation of apical filling after root canal filling by 2 different techniques. J Can Dent Assoc 75 (2009) 201a-201d.

[17] Cavenago BC, Duarte MA, Ordinola-Zapata R, Marciano MA, Carpio-Perochena AE, Bramante $\mathrm{CM}$. Interfacial adaptation of an epoxy-resin sealer and a self-etch sealer to root canal dentin using the System B or the single cone technique. Braz Dent J 23 (2012) 205-11.

[18] Ordinola-Zapata R, Bramante CM, Graeff MS, Carpio Perochena A, Vivan RR, Camargo EJ, Garcia RB, Bernardineli N, Gutmann JL, Moraes IG. Depth and percentage of penetration of endodontic sealers into dentinal tubules after root canal obturation using a lateral compaction technique: a confocal laser scanning microscopy study. Oral Surg Oral Med Oral Pathol Oral Radiol Endod 108 (2009) 450-457.

[19] Ordinola-Zapata R, Bramante CM, Bernardineli N, Graeff MS, Garcia RB, Moraes IG, Debelian G A preliminary study of the percentage of sealer penetration in roots obturated with the Thermafil and RealSeal-1 obturation techniques in mesial root canals of mandibular molars. Oral Surgery, Oral Medicine, Oral Pathology, Oral Radiology and Endodontology 108 (2009) 961-8.

[20] Keles A, Alcin H, Kamalak A, Versiani MA. Micro-CT evaluation of root filling quality in ovalshaped canals. Int Endod J 47 (2014) 1177-8. 
[21] Wolf M, Kupper K, Reimann S, Bourauel C, Frentzen M. 3D analyses of interface voids in root canals filled with different sealer materials in combination with warm gutta-percha technique. Clin Oral Investig 18 (2014) 155-61.

[22] van der Sluis LWM, Gambarini G, Wu MK, Wesselink PR. The influence of volume, type of irrigant and flushing method on removing artificially placed dentine debris from the apical root canal during passive ultrasonic irrigation. Int Endod J 39 (2006) 472-476.

[23] Boutsioukis C, Lambrianidis T, Verhaagen B, Versluis M, Kastrinakis E, Wesselink P, van der Sluis L. The Effect of Needle-insertion Depth on the Irrigant Flow in the Root Canal: Evaluation Using an Unsteady Computational Fluid Dynamics Model. J Endod 36 (2010) 1664-68.

[24] Vera J, Arias A, Romero M. Effect of maintaining apical patency on irrigant penetration into the apical third of root canals when using passive ultrasonic irrigation: an in vivo study. J Endod 37 (2011) 1276-1278.

[25] van der Sluis L, Vogels M, Verhaagen B, Macedo R, Wesselink P. Study on the Influence of Refreshment/Activation Cycles and Irrigants on Mechanical Cleaning Efficiency During Ultrasonic Activation of the Irrigant. J Endod 36 (2010) 737-740.

[26] Jiang L, Verhaagen B, Versluis M, van der Sluis L. Evaluation of a Sonic Device Designed to Activate Irrigant in the Root Canal. J Endod 36 (2010) 143-146.

[27] Loushine BA, Bryan TE, Looney SW, Gillen BM, Loushine RJ, Weller RN, Pashley D, Tay F. Setting properties and cytotoxicity evaluation of a premixed bioceramic root canal sealer. J Endod 37 (2011) 673-7.

[28] Schilke R1, Lisson JA, Bauss O, Geurtsen W. Comparison of the number and diameter of dentinal tubules in human and bovine dentine by scanning electron microscopic investigation. Arch Oral Biol. 45 (2000) 355-61.

[29] Mannocci F1, Pilecki P, Bertelli E, Watson TF. Density of dentinal tubules affects the tensile strength of root dentin. Dent Mater. 20 (2004) 293-6. 